\title{
Simple shear in 3D DEM polyhedral particles and in a simplified 2D continuum model
}

\author{
Paul Langston · Jun Ai · Hai-Sui Yu
}

Received: 28 January 2013 / Published online: 10 May 2013

(C) The Author(s) 2013. This article is published with open access at Springerlink.com

\begin{abstract}
We develop a discrete element model (DEM) simulation of mixed regular rounded polyhedra and spheres in simple shear with walls and periodic boundaries in 3dimensions. The results show reasonably realistic behaviour developing shear and dilation or compaction depending on whether the initial state is dense or loose. Similarly non-coaxiality of principal stress direction and strain rate direction are shown. Polyhedra show more general realistic behaviour than spheres but take significantly longer to run. Particle forces include normal elastic, damping, and tangential friction and rolling friction. No cohesion or interstitial fluid is modelled. A separate simplified dynamic implicit finite difference Eulerian continuum model is developed and its parameters are used to fit the DEM results. This uses mass and momentum balances, a non-linear constitutive model and Mohr-Coulomb failure criterion. It runs in 2D with periodic boundaries effectively making it pseudo-1D. The model can reproduce the general trend of the DEM results and is a good basis for further development and understanding the physics.
\end{abstract}

Keywords Simple shear · Non-coaxiality · Particle shape · Polyhedral · DEM · Continuum

\section{Introduction}

\subsection{Background}

Simple shear is one of the most common deformation patterns of particulate systems. For example, simple shear is generally

P. Langston $(\bowtie) \cdot$ J. Ai $\cdot$ H.-S. Yu

Nottingham Centre for Geomechanics, The University of Nottingham, University Park, Nottingham NG7 2RD, UK

e-mail: P.Langston@nottingham.ac.uk appreciated as the typical deformation mode in localised failure zones of granular materials and in shaking level grounds under seismic shear waves. In addition, it is also the dominant deformation mode in plane shear flow [1].

As one of the earliest methods for laboratory testing of soils, the simple shear test has significantly contributed to the initial work on the development of critical state soil mechancis at Cambridge e.g., [2]. A good review of simple shear testing of soils has been given by Airey et al. [3] where both advantages and limitations of the test are highlighted.

A key feature of simple shear loading is that the principal stress directions are not fixed but rotate during shear, resulting in a non-coaxiality between the principal stress and strain rate directions with the latter largely fixed during the simple shearing [4]. Due to anisotropy, the particle assembly may deform during principal stress rotation even when the shear stress level (or the mobilised friction angle) is kept constant. For example, it has been shown the rotation of principal stress direction has a significant effect on the permanent strain development [5]. In fact, principal stress rotations are generally involved in loading conditions encountered in geotechnical engineering. Considerable evidence from laboratory soil tests, numerical simulations and micro-mechanical research of granular materials has shown that the principal axes of stress and strain rate are generally not coincident e.g., [5-10]. This "non-coaxiality" phenomenon has an important implication on the constitutive modelling of granular materials $[11,12]$.

As a complementary tool to the experimental apparatus of simple shear, discrete element modelling (DEM) has been adopted to help investigate the simple shear behaviour of granular assembly. Most earlier DEM studies of simple shear mainly focused on steady state behaviour e.g., [1,1315]. However, such large strain regimes are less relevant to geotechnical applications as the volumetric strain has been 
fully mobilised and the directions of principal stress and strain rate have already developed to be largely coincident. In contrast, the relatively small shear strain regime is of more importance in geomechanics where deformation and modulus are important factors for practical design. Previous DEM simulations investigating non-steady regime of simple shear are largely limited to strength and volumetric behaviour e.g., [16-19]. Only very few studies consider the non-coaxiality [20-22].

Most DEM studies either use spheres in 3D or circles in 2D. The advantage of this is that the code is simpler and faster than for non-spheres and many more particles can be simulated in a study. Such studies have produced realistic and useful results in many areas. However, many granular systems comprise non-spherical grains where the shape significantly affects the bulk response. Several techniques have been applied to model non-spheres including, sphere-union, sphere-intersection, hemi-sphere-endedcylinders, super-quadrics and polyhedra. Mack et al. [23] reviews some of the methods and develops a 3D "rounded polyhedral" model which is validated against a small laboratory data of hopper flow with polyhedral dice and plastic spheres. This shows that the behaviour of the polyhedra with rounded edges is quite complex; for example in some cases they flow slightly faster than equivalent volume spheres yet can have a greater probability of forming a jammed system.

Polyhedral particles introduce additional difficulties in DEM studies, namely when defining the contact plane and the contact frictional forces between particles. Mack et al. [23] models a multi-point contact to describe the region of contact and bases the analysis on the point of largest overlap. Pena et al. [24] sets an upper limit for the integration step based on the kinetic energy decay. It also shows that the usual way of defining the contact plane between two polygonal particles is, in general, not unique which leads to discontinuities in the direction of the contact plane while particles move. A more accurate definition for the contact plane is based on the shape of the overlap area between touching particles, which evolves continuously in time. Another possibility would be to model the particles as overlapping spheres, however, this might affect the behavior.

Discrete element model has the intrinsic advantage that it can model the real system down to the individual particle level, and hence reproduce realistic assembly behaviour. It should be remembered, however, that a number of simplifications are applied at the particle level such as the use of "rolling friction" for spheres. A further level of detail can be added with the FEM_DEM approach, Latham et al. [25]. The main limitation of DEM is that only relatively small systems can be modelled. The complementary approach is continuum, for example a model of cohesionless granular flows described in Daniel et al. [26] - in which a constitutive model is developed based on the idea that friction and solids fraction are exclusive functions of the inertial number, which represents the ratio of inertial to normal forces-the model was able to represent velocity and solid volume fraction profiles in experimental systems. Jiang et al. [27] uses kinematic variables such as 'averaged micro-pure rotation rate' to bridge discrete and granular mechanics in 2D. Continuum models can simulate larger systems. They can also help our understanding of the physics in that the behaviour has to be more rigorously defined, whereas in a discrete system the behaviour arises out of the interaction of the elements in a "numerical experiment". Ideally one could say you want: field measurements, laboratory scale experiments, continuum models, DEM and even FEM_DEM models for an optimal engineering study. DEM results could help formulate constitutive and failure equations for use in continuum models.

It is more generally noted that the study of non-spherical particles can have importance beyond granular matter as new nano and micrometer sized particles with "hard" interactions have been synthesized recently [28].

\subsection{Objectives and outline of paper}

This study uses the 3D DEM polyhedral model developed in Mack et al. [23] to model a simple shear cell to investigate the micromechanical response in slow shear comparing a sample of spheres and a sample of mixed polyhedra and spheres (Sect. 2). It then develops a separate 2D continuum dynamic model fitting some of its parameters to the DEM results (Sect. 3 ). The aim is to investigate the feasibility of DEM polyhedra for such an application and investigate how it might help in the development of a complementary continuum approach. Section 4 describes the conclusions and recommendations for future work.

\section{DEM 3D polyhedral shear model}

\subsection{Model summary}

Mack et al. [23] describes an algorithm for particle-particle contact calculation for polyhedra with rounded edges. This was applied with standard DEM techniques to model granular flow in hoppers. A "softened" normal spring particle contact model was used along with linear contact damping and tangential spring-slider for friction. The model was tested against laboratory experiments on polyhedral dice and plastic spheres. Individual particle properties were measured beforehand, such as dimensions, density, friction and damping. The model results showed very good agreement to experimental results for packing structure, rate of discharge and qualitatively nature of flow. It is emphasised that no model parameters were fitted to give a match. The model also includes 
Fig. 1 a Snapshot of particles in shear cell for mix polyhedral and spheres sample (only particles near walls shown). b Snapshot of particles in shear cell for all spheres sample. Left general view direction (only particles near walls shown); Right front view of all spheres. More efficient packing for spheres results in slightly smaller cell than for mix polyhedra and spheres (a)
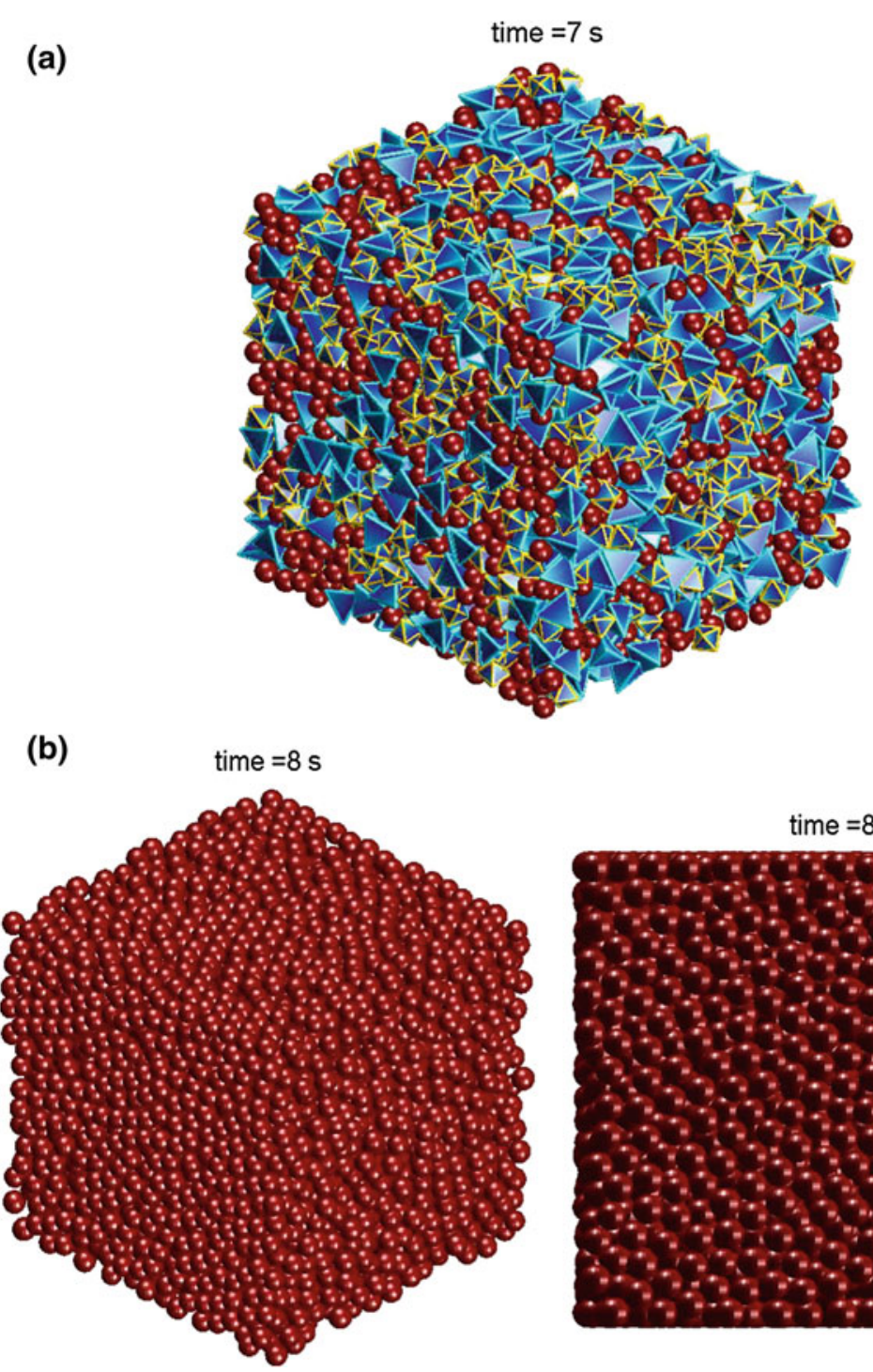

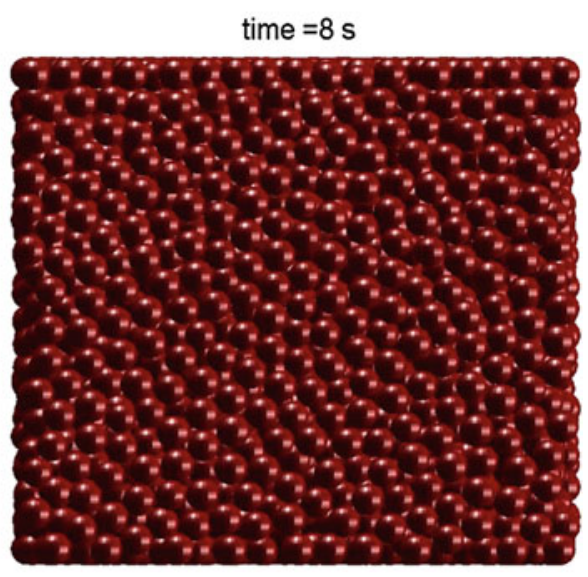

a simple cohesion model but this was not tested experimentally. The current study does not consider particle cohesion.

\subsection{DEM shearing scenarios}

Four samples are modelled:

1. Mix polyhedra and spheres in initial dense state

2. All spheres in initial dense state

3. Mix polyhedra and spheres in initial loose state

4. All spheres in initial loose state

The mixed sample comprises equal numbers of tetrahedral, octahedral and spherical particles all with the same volume and material. The polyhedra have rounded edges and vertices. The shear cell is approximately cubic, with fixed periodic boundaries in $x$ (horizontal) the direction of shear. A constant vertical force is applied on the bottom movable wall. The top wall has a fixed $z$ position and shears right to left $(-x)$; the bottom wall moves vertically in $z$ and shears left to right; the front and back walls are fixed; the bottom movable wall mass is approximately 0.2 total particle mass; all particle-wall interactions have friction. Figure 1 shows some example configurations of particles in the shearing cell.

The scenario comprises a preparation stage followed by shear. Preparation comprises normal compression of initially non-contacting particles, with zero shear to create a sample with the required state-Fig. 2. Gravity is zero throughout. The normal load during preparation can be different to that during shear. On commencement of shear the top and bottom walls are given a constant tangential velocity to achieve the required shear rate. Note that even though gravity is zero the $x y z$ directions are significant in identifying the stress components in relation to shear direction and the different nature of the boundaries: fixed, movable or periodic. 

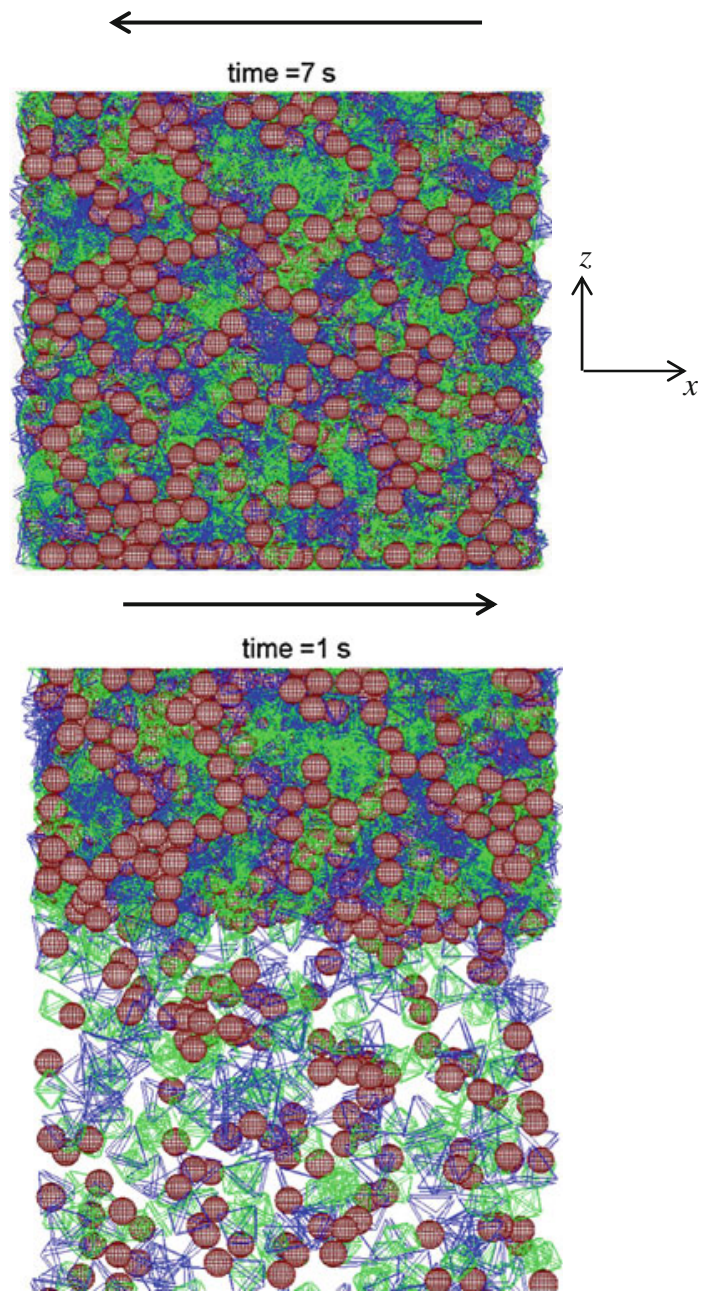

Fig. 2 View of particles from front of polyhedra mix ( $x$ horizontal, $z$ vertical) with periodic boundaries right and left, shearing walls top and bottom, fixed walls front and back. Bottom figure shows part of cell during preparation stage before shearing as bottom wall is raised. All particles are given initial upward velocity equal to wall vertical velocity; no gravity (wire frame particle graphics)

Our study focuses on the quasi-static flow regime. The simple shear rate is controlled to limit the inertial number $I$ to prohibit potential inertial effects. The inertial number is a dimensionless measure defined as the ratio between a micro timescale $T_{P}$ and a macro timescale $T_{\dot{\gamma}}$ [1]. It is defined in our study as:

$I=\frac{T_{P}}{T_{\dot{\gamma}}}=\frac{d \sqrt{\rho / P}}{1 / \dot{\gamma}}$

where $d, \rho, P$ and $\dot{\gamma}$ are mean particle diameter, assembly bulk density, mean stress and simple shear strain rate respectively. A flow system approaches quasi-static as $I \ll 1$. In our simulations the shear rate is approximately constant and $I$ is approximately $7.0 \times 10^{-4}$, similar to some other studies e.g., [15], and so can be considered quasi-static.
Particle contact forces modelled are as described in Sect. 2.1 above. The assembly stress and strain rate are periodically sampled in a spherical region around the centre of the cell.

The stress tensor $\bar{\sigma}_{i j}$ across the whole cell is obtained by averaging the stress tensor of each particle $\bar{\sigma}_{i j}^{(k)}$ over the $N_{p}$ particles contained in the cell [29]

$\bar{\sigma}_{i j}=\frac{1}{V} \sum_{N_{p}} \bar{\sigma}_{i j}^{(k)} V^{(k)}$

where $V$ and $V^{(k)}$ are the total volume of the cell and the volume of particle $k$ respectively. The stress tensor of each particle was calculated from the contact forces $f_{i}^{(c)}$ acting on the particle

$\bar{\sigma}_{i j}^{(k)}=\frac{1}{V^{(k)}} \sum_{N_{c}}\left(x_{i}^{(c)}-x_{i}^{(k)}\right) f_{j}^{(c)}$

where $N_{c}$ is the number of contacts; $x_{i}^{(c)}$ and $x_{i}^{(k)}$ are the locations of contact point and particle centroid, respectively.

The strain rate tensor $\dot{\varepsilon}_{i j}$ is obtained by evaluating the velocity gradient tensor of the deforming particle assembly [29]. This is achieved via a least-squares best-fit that minimizes the error $\delta$ between the predicted and measured relative velocities of all particles within the measurement domain:

Table 1 Principal data in DEM simulation

\begin{tabular}{|c|c|}
\hline DEM parameter & Value \\
\hline Number of particles & 5,000 \\
\hline $\begin{array}{l}\text { Tetrahedron "radius" }(\mathrm{cm}) \text {, } \\
\text { all particles same mass } \\
\text { and volume }\end{array}$ & 0.5 \\
\hline Particle rounded edge radius $(\mathrm{cm})$ & 0.05 \\
\hline $\begin{array}{l}\text { Shear cell (cubic) approximate } \\
\text { dimensions }(\mathrm{cm})\end{array}$ & 8.5 (mix); 8 (spheres) \\
\hline $\begin{array}{l}\text { External load sample initial } \\
\text { preparation }(\mathrm{kPa})\end{array}$ & 15 (dense); 5 (loose) \\
\hline External load during shear $(\mathrm{kPa})$ & 5 \\
\hline Shear rate $\left(\mathrm{s}^{-1}\right)$ & 0.1 approx. \\
\hline $\begin{array}{l}\text { Times: load change in } \\
\text { preparation, shear } \\
\text { commence }(\mathrm{s})\end{array}$ & $2.5,3.0$ \\
\hline $\begin{array}{l}\text { Sample spherical region } \\
\text { size located at centre }\end{array}$ & 0.8 of cell dimension \\
\hline Cohesion factor & 0 \\
\hline Particle solid density $\left(\mathrm{kg} / \mathrm{m}^{3}\right)$ & 2,600 \\
\hline Particle coefficient of friction & 0.6 \\
\hline Particle-wall friction & 0.9 \\
\hline Particle damping & Moderate \\
\hline Rolling friction coefficient & 0.005 \\
\hline
\end{tabular}


Fig. 3 Example of particle-particle contact force vectors in region of vessel during shear. Vectors drawn particle centre to centre, thickness proportional to magnitude. Top wall is moving right to left. Vector orientation in $x z$ plane distribution is shown
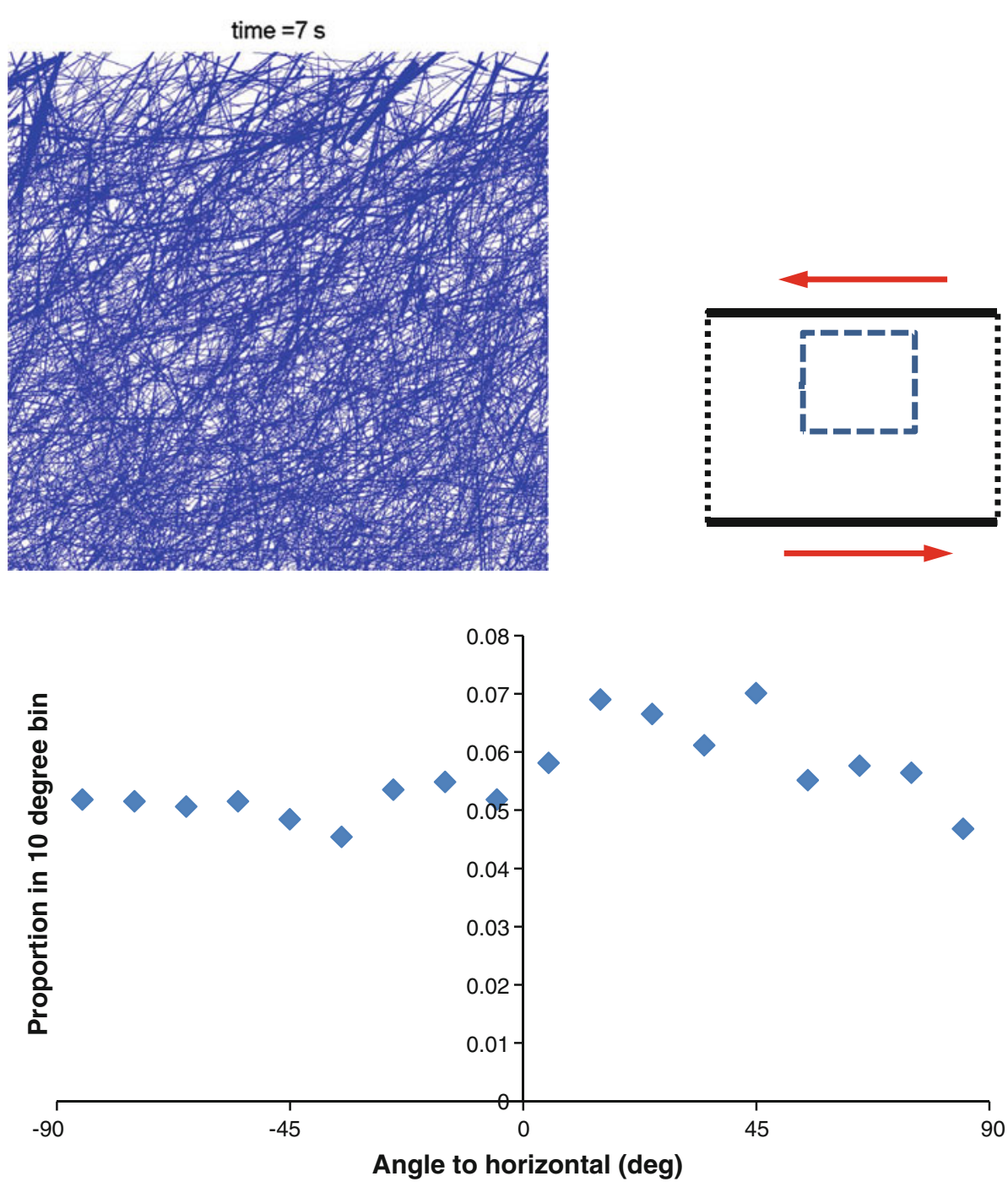

$$
\begin{aligned}
\delta & =\sum_{N_{p}}\left|\tilde{v}_{i}^{(k)}-\tilde{V}_{i}^{(k)}\right|^{2} \\
\frac{\partial \delta}{\partial \dot{\varepsilon}_{i j}} & =0
\end{aligned}
$$

where $\tilde{v}_{i}^{(k)}$ and $\tilde{V}_{i}^{(k)}$ are respectively the predicted (product of strain rate tensor and relative position) and measured (DEM value) relative velocities of particle $k$ against the mean particle velocity of the monitored assembly.

It is noted that the all sphere sample, which has the same number and mass as the mixed sample, has a closer packing hence the shear cell for the spheres is slightly smaller. Periodic boundaries are used to avoid deformation of the cell in shear. This aspect is discussed later with the results. Experimental simple shear cells typically utilise rough top and bottom platens, but smooth and rigid side walls in order to allow the free dilation of the confined solid in vertical direction. Such different boundary wall conditions lead to incompati- ble shear stress developed and hence non-uniform stress field in the solid. While using periodic boundaries, the side walls in the shearing direction are removed so that the incompatible boundary shear stress issue are avoided. It would also have been possible to set up a more idealised system using periodic boundaries for the front and back as well or used frictionless walls here. The system used here is more representative of a practical shear box. Note also that the external load is held constant, but the internal stress in $z$ can vary dynamically as it would in a practical system.

The principal data used in the DEM simulations is shown in Table 1.

Scenario for dense state:

1. Normal compression under high external load and zero particle friction, no shear

2. Normal compression continues under load used for shear and particle friction introduced, no shear

3. Shear commences 

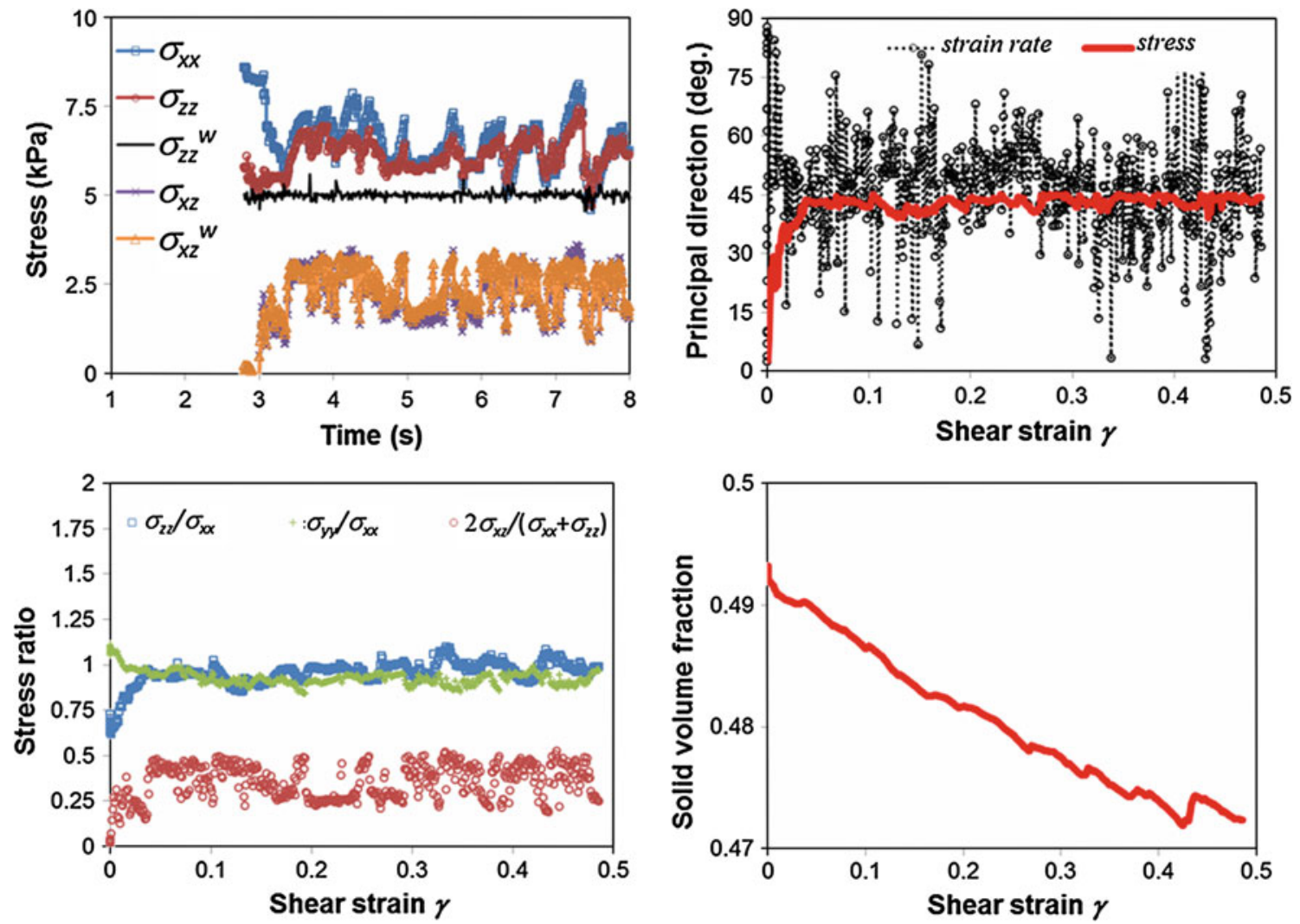

Fig. 4 DEM results: mix polyhedra with high external load in sample preparation: stress components, results are not shown before $t=2.8 \mathrm{~s}$ while sample in preparation; major principal stress and strain rate orientations relative to $x$-axis; stress ratios; solid volume fraction

Scenario for loose state:

1. Normal compression under load used for shear and with particle friction, no shear

\section{Shear commences}

Wall friction is set high to help impart shearing.

\subsection{DEM results}

Figure 3 shows a view of the contact force vectors in a region of the shear cell for a mixed sample case. There is a clear indication of the principal stress direction. The figure also shows the distribution of the contact vectors' angles in the $x z$ plane supporting this, but the main quantitative demonstration is in the principal major stress direction shown in the results following. The results of the DEM simulations for the four samples are shown in Figs. 4, 5, 6 and 7.

Figure 4 shows the mixed dense state case. The sample is not quite at steady-state before shear commences, but close enough - see figure top left, there is about $1 \mathrm{kPa}$ variation in the internal stress components for time $2.8-3 \mathrm{~s}$, but the wall stresses are near constant. There are quite a lot of fluctuations in internal stress as would be expected on equivalent laboratory measurements and in major principal strain rate direction. Fluctuations in wall normal stress are much lower. Ideally more particles should be modelled, but 5,000 seems to give overall reasonable results. Computer runtime is currently the main restriction on modelling more polyhedra. The major principal strain rate direction fluctuation is due to localised rearrangement of particles as they move around each other. This has also been shown in our other work on 2D shear of spheres (not yet published), which also would explain stress fluctuation. There are good indications of dense packing in the shear stress response and solid volume fraction evolution showing dilation. The internal normal stresses are isotropic in the $x-y$ horizontal plane (see stress ratio) which gives some indication that the periodic boundary is not significantly influencing the system. It has not achieved critical state in shear time simulated as shown by the solid volume fraction, but a significant proportion of the transient response is shown. Wall shear and internal shear stresses are in very close agreement, but it is noted that the internal normal stress in $z$ is slightly higher than the wall stress. The horizontal stress is initially higher than vertical $\left(K_{0}=\left.\left(\sigma_{x x} / \sigma_{z z}\right)\right|_{t=3 s}>1\right)$, which is reasonable given the preparation stage. The internal normal stresses approach each other hence the major principal stress direction rises as expected to near $45^{\circ}$. It is noted that because this is a $3 \mathrm{D}$ simple shear scenario, the major principal stress might not 

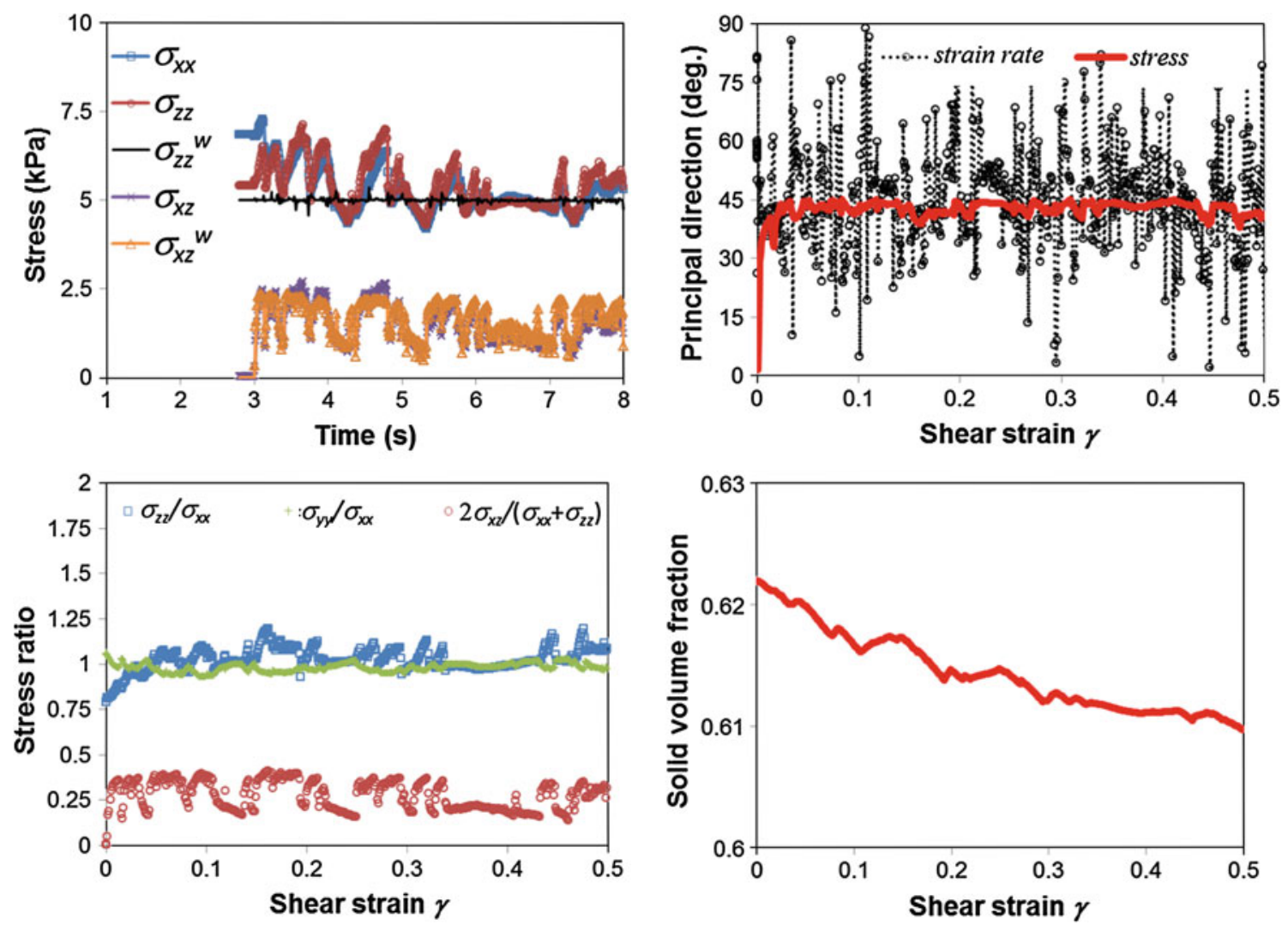

Fig. 5 DEM results: spheres with high external load in sample preparation

always lie in the $x-z$ plane, so in terms of non-coaxiality, it is emphasised that we are concerned with the $x-z$ plane components. The directions are relative to horizontal $x$ direction.

Figure 5 shows the spheres dense state case. This case is fully settled before shear. The general response in shear is similar in trend to the mixed particle case above showing dense phase behaviour, but with significant differences. In terms of stresses the internal stresses are similar to the wall stresses. The shear stress (and shear stress ratio) is lower than with polyhedra showing less frictional behaviour as would be expected with spheres. The major principal stress direction approaches $45^{\circ}$ more quickly. The major principal strain rate direction fluctuates about $45^{\circ}$ as for the polyhedra-this is very dependent on instantaneous localised motion, even if the motion is less. As expected there is significantly closer packing than with polyhedra (hence the smaller shear cell) and less dilation. Given that most granular systems do not comprise only near spherical particles the polyhedra resultsalthough somewhat idealised here and with some spheresare more realistic, however, the simulation takes about 4-5 times longer which is significant. More work is needed to improve model efficiency.

Figure 6 shows the mixed loose state case. There are indeed indications of loose behaviour with some compaction on commencement of shear and generally rising mean shear stress. $K_{0}<1$ here which is reasonable and the major principal stress direction decreases from $90^{\circ}$ but changes more slowly than for the dense case. This is also consistent with the slower changing stresses and somewhat lower strain rate direction fluctuation. It is noticeable that the mean of the strain rate direction changes during shear and rises to $45^{\circ}$ and that the mean of the major principal stress direction does not reach $45^{\circ}$. The amount of compaction shown in the solid volume fraction is similar to the amount of dilation for the dense polyhedra.

Figure 7 shows the spheres loose state case. This also exhibits somewhat loose behaviour. Generally the differences between the all spheres case here and the previous mixed particle loose case are similar to the differences shown in the corresponding dense cases. It is noticeable here that the major principal stress direction reaches about $55^{\circ}$.

\section{Continuum 2D shear model}

\subsection{Model summary}

It is emphasised that this is a simplified model and should be considered as an initial attempt to match the more detailed DEM with a continuum approach. 

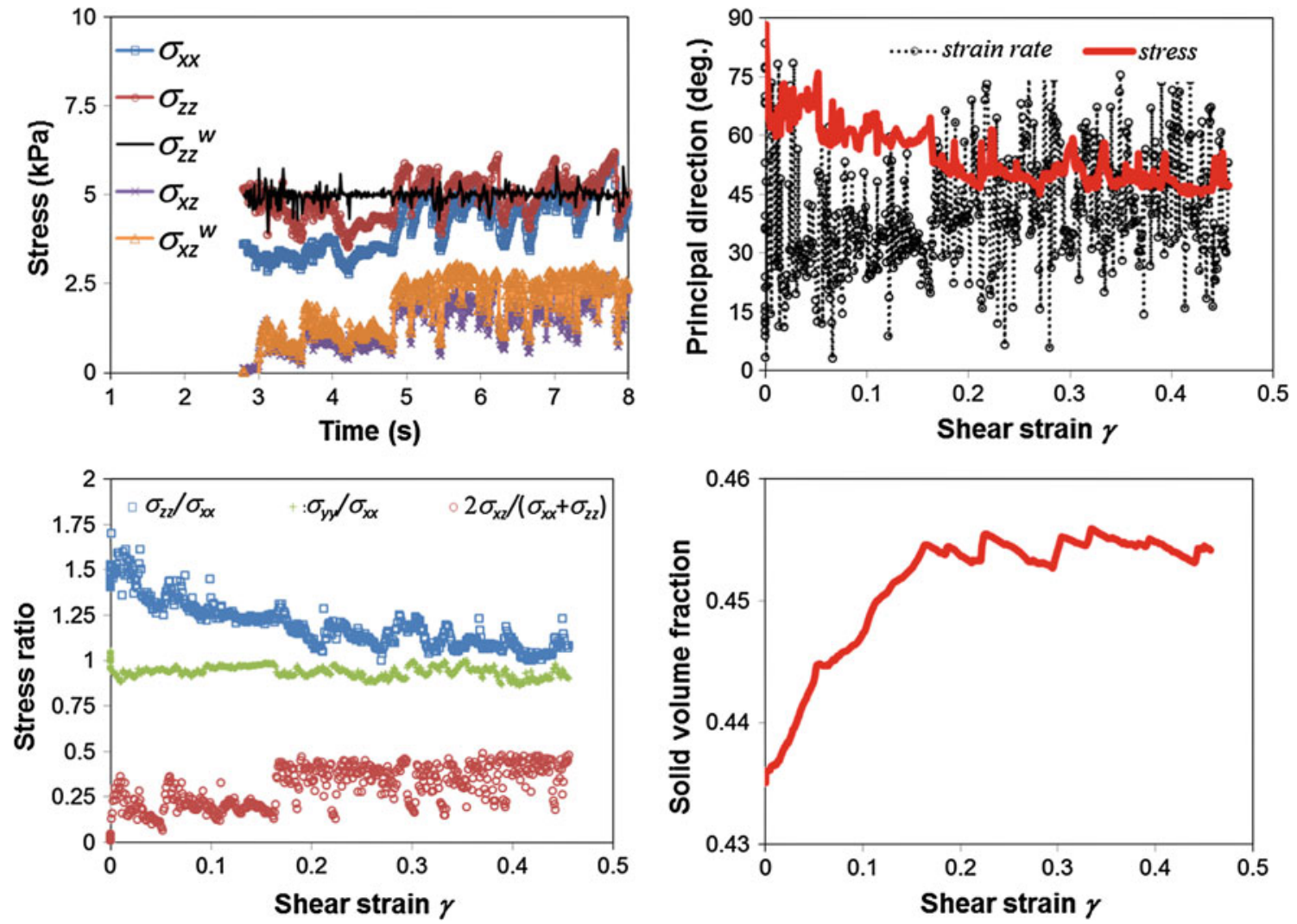

Fig. 6 DEM results: mix polyhedra with low external load in sample preparation
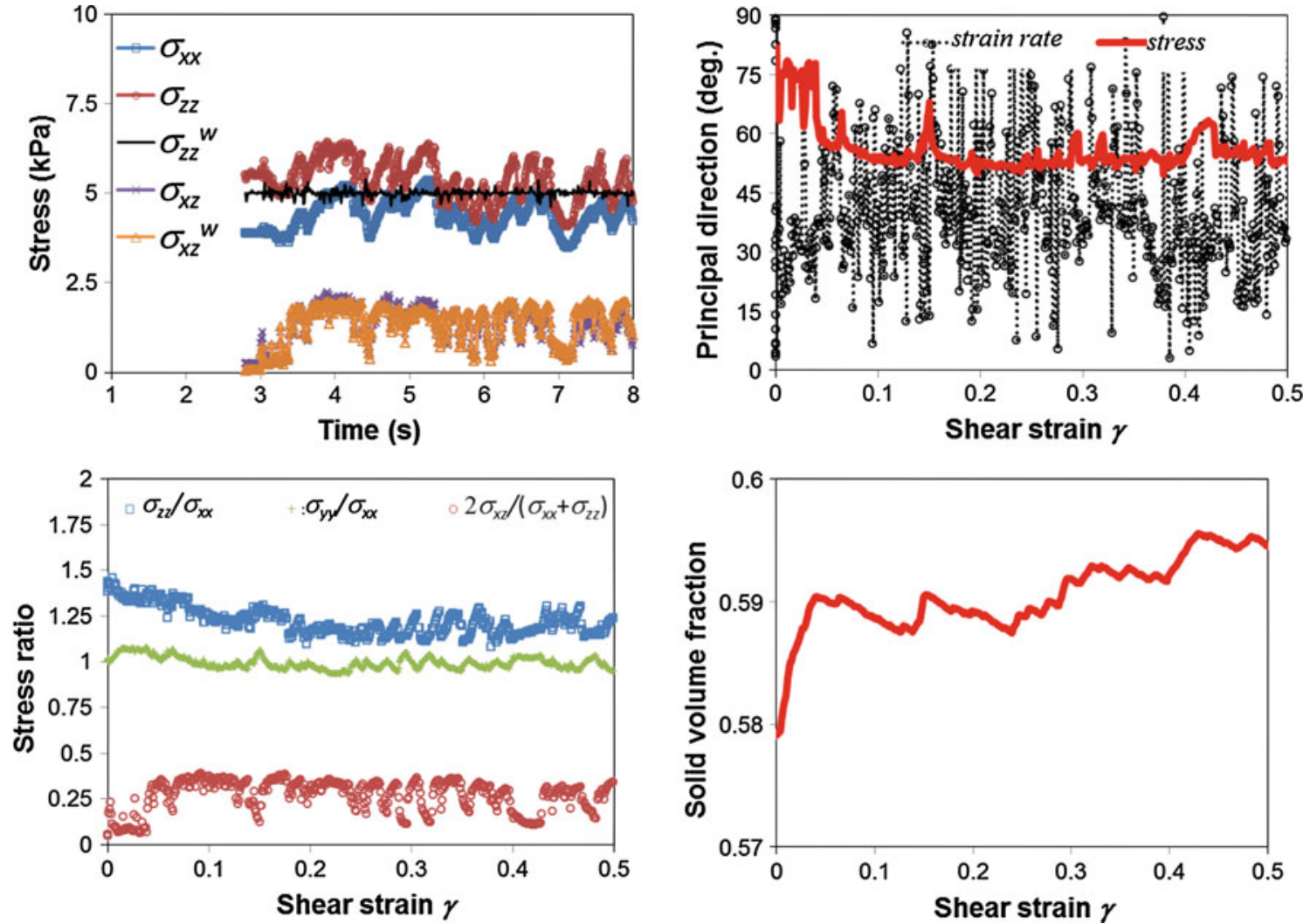

Fig. 7 DEM results: spheres with low external load in sample preparation 
Table 2 Principal data in 2D continuum simulation

\begin{tabular}{ll}
\hline Continuum model parameter & Value \\
\hline Shear cell height $(\mathrm{m})$ & 1.1 \\
Sub-cell length $(\mathrm{m})$ & 0.1 \\
$\begin{array}{l}\text { Sample region-all cells } \\
\quad \text { except boundary cells }\end{array}$ & Cell rows $2-10$ \\
External load during shear $(\mathrm{kPa})$ & 5 \\
Shear rate $\left(\mathrm{s}^{-1}\right)$ & 0.1 \\
Times: shear commence (to & 3.0 \\
$\quad$ facilitate DEM & \\
$\quad$ comparison) $(\mathrm{s})$ & 50 \\
Young's modulus $(\mathrm{kPa})$ & 0.3 \\
Poisson's ratio & 0 \\
Cohesion $(c)$ & 2600 \\
Particle solid density $\left(\mathrm{kg} / \mathrm{m}^{3}\right)$ & 0.4 for polyhedral cases \\
Internal coefficient of friction & \\
Damping proportional factor & \\
Constitutive parameter $\left(n_{s t r}\right)^{\mathrm{a}}$ & 0.1 \\
Strain rate factors $\left(\chi_{x}, \chi_{z}\right)^{\mathrm{a}}$ & 1.5 \\
Initial stress and stress ratio $\left(K_{0}\right)$ & 0,9 \\
${\text { Initial solid volume fraction }\left(\eta_{0}\right)}_{\text {Critical solid volume fraction }\left(\eta_{c}\right)}^{\text {Set by DEM results }}$ \\
\hline
\end{tabular}

a Trial and error was used to estimate general suitable values

The shear cell is divided into square sub-cells. It is an Eulerian fixed grid ( $x$-horizontal, $z$-vertical) with staggered grids for stress and velocity. Left and right are periodic boundaries hence conditions are constant along $x$. (This phenomenon also helps to detect bugs in the code.) In effect this makes it pseudo 1-dimensional, although there are velocities in $x$ and $z$. The walls are not explicitly modelled but shear is imposed by setting a constant velocity $x$ in the top (+ve) and bottom ( $-\mathrm{ve})$ rows at start of shear; the shear rate is specified by the user. All velocities are initialised to zero.

Mass and momentum balances are applied to granular flow in each sub-cell using an implicit solution of velocity at end of the time-step with relaxation of stress to get convergence. It is a single phase (solid no fluid) but with solid volume fraction $\eta$ applied-stresses are bulk; gravity is zero. The momentum balance can be written:

$\frac{\partial(\eta \rho \bar{u})}{\partial t}+\nabla(\eta \rho \overline{u u})=-\nabla \overline{\sigma_{s}}-\nabla \overline{\sigma_{d}}$

where $\rho$ is solid density, $u$ is velocity, subscripts $s$ indicate static and $d$ damping.

The initial conditions are set as follows: zero shear and velocity; specify $\sigma_{x x}, \sigma_{z z}\left(K_{0}\right)$, hence estimate $\varepsilon_{x x}, \varepsilon_{z z}$. Then allow the system to settle to steady state before shear is imposed as described above. It would be possible to model constant volume and mass during shear, but here constant external loading during shear is modelled by allowing mass to flow in/out of the top cells (ie constant volume but not constant mass in the system).

Strain rate is calculated from the velocities at mid-time step. The strain increment is then calculated:

$\overline{\Delta \varepsilon}=\overline{\varepsilon_{r}} \Delta t$

A further component of strain increment is added as shown below and similarly for the $z z$ component. This is somewhat speculative and is necessary to model particulate like response. $\chi$ represents two constants in $x$ and $z$.

$\Delta \varepsilon_{x x}^{\prime}=\left(\eta-\eta_{c}\right) \chi_{x} \Delta \varepsilon_{z x}$

The stress increment is then calculated from the strain increment using Young's modulus $E$ and Poisson's ratio $v$. A non-linear form of $E$ is used, where $\eta$ is solid volume fraction, $\eta_{c}$ is a critical value constant, $n_{s t r}$ is a constant.

$$
\begin{aligned}
E^{*} & =E \eta_{r}^{n_{s t r}} \\
\eta_{r} & =\eta / \eta_{c}
\end{aligned}
$$

The system response is sensitive to the failure criterion. In this initial model the Mohr-Coulomb criterion is used, $\sigma_{m}$ is mean normal stress, $r_{m c_{-} \max }$ is maximum radius of Mohrcircle, $\phi$ is constant friction angle, $c$ is constant cohesion ( $c=0$ in this study). If in a cell the stress state implies that the radius of Mohr's circle is greater than the maximum, then it is reduced keeping the mean normal stress constant. (Another variant on this was tested keeping the minor principal stress constant, but that gave unrealistic results.)

$r_{m c_{-} \max }=\sin (\phi)\left(\sigma_{m}+c / \tan (\phi)\right)$

A viscous (damping) shear stress is also applied directly proportional to shear rate as function of $E$ and $v$. This is necessary to stop oscillations in velocity and stress.

\subsection{Shear scenario}

Table 2 shows the principal data used in the continuum model and Fig. 8 shows a snapshot of the shear cell configuration and graphical representation of solid volume fraction and velocity in each sub-cell. The data has been set as close as possible to the DEM cases. The length scale is somewhat different but that is not significant.

\subsection{Continuum results}

The results of the continuum simulations are only considered here for the mixed polyhedra cases for the dense and loose samples and are shown in Figs. 9 and 10. The format of the results figures corresponds to the DEM figures, except that there is no wall stress or stress in $y$. Note the system 
Fig. 8 Continuum model simple shear cell representation snapshot. Each circle represents square sub-cell within system. Periodic boundaries left and right making values in each column the same i.e., the system becomes pseudo 1-dimensional but with velocity in $\mathrm{x}$ and $\mathrm{z}$. Circle size is proportional to $\eta$ in sub-cell, velocity is proportional to line shown, $u_{x}=0.05 \mathrm{~ms}^{-1}$ at top

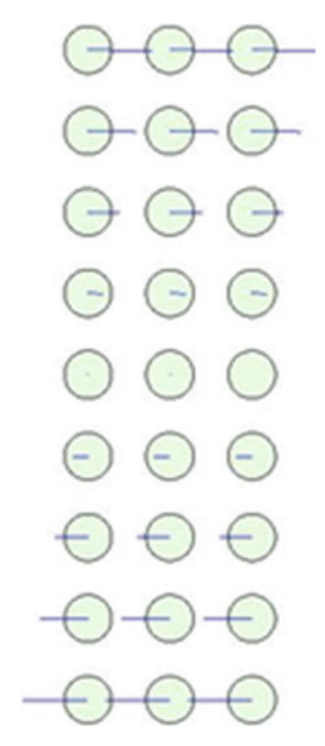

is sheared for slightly longer than DEM to display useful results, but started at $t=3 \mathrm{~s}$ for compatibility—shear rate is matched in each case.

Figure 9 shows the continuum results matched to the DEM polyhedra dense case in Fig. 4. As expected they are much smoother here due to the intrinsic nature of the continuum model. Generally there is a reasonable fit with the stresses using the reduced friction coefficient, indicating particle friction is not fully engaged in DEM; there is slightly slower dilation; major principal stress direction is similar but with a slower approach to the major principal strain rate direction. The vertical stress rises on commencement of shear then falls which is reasonable.

Figure 10 shows the continuum results matched to the DEM polyhedra loose case in Figure 6. There is a generally reasonable fit again with the reduced internal friction coefficient. The normal stresses converge slightly faster in the continuum model unlike the dense case comparison. Shear is developed in continuum faster than in DEM. Compaction is a bit slower in continuum as with the dilation in the dense case. It is considered that the DEM probably does not develop shear until the compaction has occurred.

In summary the continuum results match the DEM reasonably but the continuum is smoother, it needs a reduced friction factor and there are some differences in response speed dependent on packing fraction. Hence the model needs some further development, for example, friction angle is probably a function of packing density.
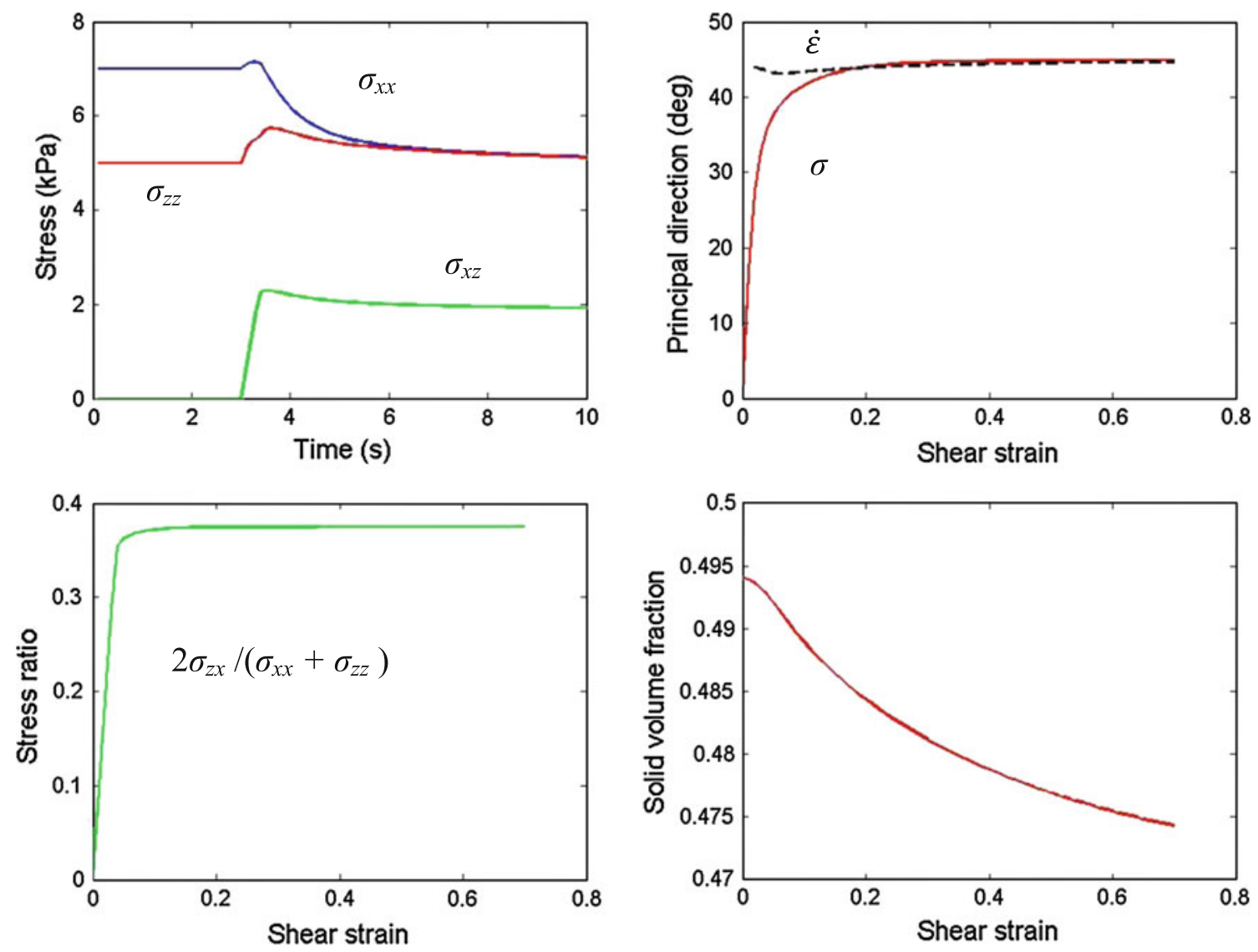

Fig. 9 Continuum results: fit to polyhedra mix dense state with $\mu=0.4, \eta_{0}=0.494, \eta_{c}=0.470, K_{0}=1.4$ : stress components; major principal stress and strain rate orientations; stress ratio; solid volume fraction 

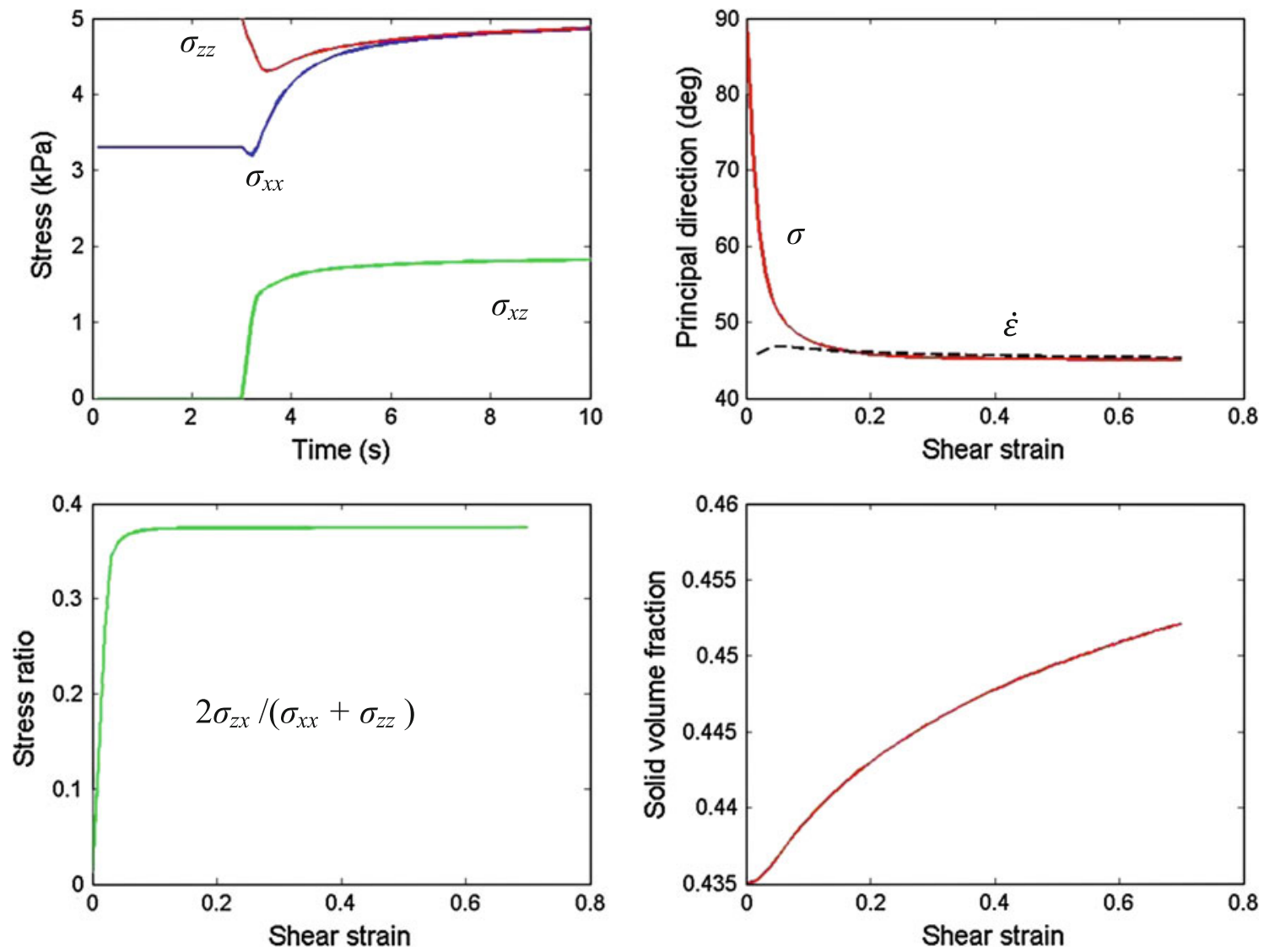

Fig. 10 Continuum results: fit to polyhedra mix loose state with $\mu=0.4 \eta_{0}=0.435, \eta_{c}=0.457, K_{0}=0.66$

\section{Conclusions}

A 3D rounded-polyhedral DEM-previously validated in experiments on non-cohesive hopper flow-is used here to monitor granular stress states in simple shear in a cubic cell with periodic boundaries with 5,000 particles. The results are generally reasonable but ideally more particles are required given the level of fluctuation. Dense and loose samples are simulated with appropriate responses in normal and shear stress, non-coaxiality and compaction or dilation.

A comparable sample of polydisperse spheres is also modeled. The polyhedra mix (including some spheres) develops higher shear stress and is more realistic of general granular systems, but takes longer to run. More work is required to develop efficiency in the simulation. It has been suggested that graphic processing units— enhanced models could help e.g., [30].

An initial continuum 2-dimensional shear cell model is developed using mass and momentum balances on Eulerian sub-cells. Data from the DEM model is used to set boundary conditions. Other model parameters are used to match the general behavior of DEM. Reasonable fits are possible showing the continuum model has appropriate features. As expected the DEM results include fluctuations due to localized sliding and rotation of particles around each other and the limited number of particles, whereas the continuum results show smooth responses.

Developing the continuum model helps to understand the granular behavior because the overall physics must be explicitly stated, whereas discrete models reproduce the overall response from individual particle physics in numerical simulations. This study should also help develop more detailed continuum models for predictions on a larger scale not currently feasible in DEM. It is envisaged that some of the model parameters used here, such as friction angle, are functions of packing density.

Acknowledgments We acknowledge the funding from Engineering and Physical Sciences Research Council (Grant EP/H011951/1) and reviewers' suggestions for model development.

Open Access This article is distributed under the terms of the Creative Commons Attribution License which permits any use, distribution, and reproduction in any medium, provided the original author(s) and the source are credited. 


\section{References}

1. MiDi, G.D.R.: On dense granular flows. Eur. Phys. J. E 14(4), 341-365 (2004)

2. Roscoe, K.: The influence of strains in soil mechanics. Geotechnique 20(2), 129-170 (1970)

3. Airey, D.W., Budhu, M., Muir-Wood, D.: Some aspects of the behaviour of soils in simple shear. Paper presented at the developments in soil mechanics and foundation engineering, New York, USA

4. Yu, H.S.: Non-coaxial theories of plasticity for granular materials. Keynote paper presented at the the 12th international conference of international association for computer methods and advances in geomechanics (IACMAG), Goa, India, 1-6 October

5. Ishihara, K., Towhata, I.: Sand response to cyclic rotation of principal stress directions as induced by wave loads. Soils Found. 23(4), 11-26 (1983)

6. Roscoe, K., Bassett, R., Cole, E.: Principal axes observed during simple shear of a sand. In: Proceedings of Geotechnical Conference, Oslo, pp. 231-237 (1967)

7. Drescher, A., de Josselin de Jong, G.: Photoelastic verification of a mechanical model for the flow of a granular material. J. Mech. Phys. Solids 20(5), 337-351 (1972)

8. Oda, M., Konishi, J.: Rotation of principal stresses in granular material during simple shear. Soils Found. 14(4), 39-53 (1974)

9. Christoffersen, J., Mehrabadi, M., Nemat-Nasser, S.: A micromechanical description of granular material behavior. J. Appl. Mech. 48, 339 (1981)

10. Gutierrez, M., Ishihara, K., Towhata, I.: Flow theory for sand during rotation of principal stress direction. Soils Found. 31(4), 121-132 (1991)

11. Hill, R.: The Mathematical Theory of Plasticity. Oxford University Press, Oxford (1950)

12. Yu, H.S.: Plasticity and Geotechnics. Springer, Berlin (2006)

13. Shen, H.H., Sankaran, B.: Internal length and time scales in a simple shear granular flow. Phys. Rev. E Stat. Nonlin. Soft Matter Phys. 70(5), 051308 (2004)

14. da Cruz, F., Emam, S., Prochnow, M., Roux, J.N., Chevoir, F.: Rheophysics of dense granular materials: discrete simulation of plane shear flows. Phys. Rev. E 72(2), 021309 (2005)

15. Sun, J., Sundaresan, S.: A constitutive model with microstructure evolution for flow of rate-independent granular materials. J. Fluid Mech. 682, 590-616 (2011). doi:10.1017/jfm.2011.251

16. Jiang, M., Yu, H.S.: Application of discrete element method to geomechanics. Mod. Trends. Geomech. 106, 241-269 (2006)
17. El Shamy, U., Gröger, T.: Micromechanical aspects of the shear strength of wet granular soils. Int. J. Numer. Anal. Methods Geomech. 32(14), 1763-1790 (2008)

18. Katagiri, J., Matsushima, T., Yamada, Y.: Simple shear simulation of 3D irregularly-shaped particles by image-based DEM. Granul. Matter 12(5), 491-497 (2010)

19. Wang, J., Yu, H., Langston, P., Fraige, F.: Particle shape effects in discrete element modelling of cohesive angular particles. Granul. Matter 13(1), 1-12 (2011)

20. Zhang, L.: The Behaviour of Granular Materials in Pure Shear, Direct shear and simple shear. Aston University, Birmingham, UK (2003)

21. Thornton, C., Zhang, L.: A numerical examination of shear banding and simple shear non-coaxial flow rules. Philos. Mag. 86(21-22), 3425-3452 (2006)

22. Wang, J.: Mechanical Behaviour of Granular Materials in Simple Shear Test Using DEM. University of Nottingham, Nottingham, UK (2009)

23. Mack, S., Langston, P., Webb, C., York, T.: Experimental validation of polyhedral discrete element model. Powder Technol. 214(3), 431-442 (2011). doi:10.1016/j.powtec.2011.08.043

24. Pena, A.A., Lind, P.G., Herrmann, H.J.: Modeling slow deformation of polygonal particles using DEM. Particuology 6(6), 506-514 (2008). doi:10.1016/j.partic.2008.07.009

25. Latham, J.P., Munjiza, A., Garcia, X., Xiang, J., Guises, R.: Threedimensional particle shape acquisition and use of shape library for DEM and FEM/DEM simulation. Miner. Eng. 21(11), 797-805 (2008)

26. Daniel, R.C., Poloski, A.P., Eduardo Sáez, A.: A continuum constitutive model for cohesionless granular flows. Chem. Eng. Sci. 62(5), 1343-1350 (2007). doi:10.1016/j.ces.2006.11.035

27. Jiang, M., Yu, H.-S., Harris, D.: Kinematic variables bridging discrete and continuum granular mechanics. Mech. Res. Commun. 33(5), 651-666 (2006)

28. Damasceno, P.F., Engel, M., Glotzer, S.C.: Predictive self-assembly of polyhedra into complex structures. Science 337(6093), 453-457 (2012). doi:10.1126/science.1220869

29. Itasca: Manual of Particle Flow Code (PFC2D/3D). Itasca Consulting Group (2008)

30. Anderson, J.A., Lorenz, C.D., Travesset, A.: General purpose molecular dynamics simulations fully implemented on graphics processing units. J. Comput. Phys. 227(10), 5342-5359 (2008). doi:10. 1016/j.jcp.2008.01.047 